\section{Diagnosis of biliary atresia can not be excluded by declining trend of serum direct bilirubin}

\author{
Keita Terui, 1 Yasuyuki Higashimoto,2 \\ Eriko Saito, ${ }^{3}$ Takeshi Saito, ${ }^{1}$ \\ Tetsuya Mitsuanga,1 Mitsuyuki Nakata, \\ Jun Iwai,2 Hideo Yoshida1 \\ 1Department of Pediatric Surgery, \\ Graduate School of Medicine, Chiba \\ University; 2Department of Pediatric \\ Surgery, Chiba Children's Hospital; \\ 3Department of Pediatric Surgery, \\ Matsudo City Hospital Children's Medical \\ Center, Japan
}

\section{Abstract}

The aim of this paper is to investigate the clinical courses of patients with biliary atresia (BA) during neonatal period. We examined 19 patients with BA, who underwent blood tests including direct bilirubin (D-Bil) within 20 days of age, in 3 tertiary hospitals in Japan. The first blood sample was collected at $8.4 \pm 6.5$ days of age. The acholic stool was observed within 2 weeks of age in 16 cases (84.2\%). Decrease of T-Bil was observed in all the subjects, with a range of reduction of $6.5 \pm 3.3$ $\mathrm{mg} / \mathrm{dL}$, from $10.4 \pm 7.5$ to $29.8 \pm 9.1$ days of age. Decrease of D-Bil was also observed in 17 out of 19 cases (89\%), with a range of reduction of $1.1 \pm 1.0 \mathrm{mg} / \mathrm{dL}$, from $15.5 \pm 8.0$ to $24.9 \pm 9.6$ days of age. A significant decrease of D-Bil was observed in 2 cases of biliary atresia splenic malformation syndrome. We therefore conclude that clinicians treating icteric infants should not exclude a diagnosis of BA even if the level of D-Bil has a declining tendency.

\section{Introduction}

Biliary atresia (BA) is an idiopathic inflammatory obliterative cholangiopathy of neonates, leading to progressive biliary cirrhosis. ${ }^{1}$ The early surgery within 60 days of age is known as one of the biggest factors which affects long-term prognosis. Therefore, early detection of infants of BA has been important issues, but both cost-effective and sufficiently efficient screening system has not been developed yet. ${ }^{2}$ According to Japanese BA Registry data, ratio of patients who underwent surgery within 60 days of age was not improving enough (31.6\% in $1990,45.2 \%$ in 2006). ${ }^{3,4}$ In US, the median age at diagnosis trended later (48.5 days between 1990 and 1994, 69.0 days between 2000 and 2004). ${ }^{5}$
Meanwhile, some cases are found in the neonatal period due to various reasons. Japanese BA Registry (1989-2006) had 116 out of 2130 cases (5.4\%) who underwent surgery within 30 days of age. ${ }^{4}$ In registry data in US (1976-1989, $n=816), 17 \%$ of cases underwent surgery within 30 days of age. ${ }^{6}$ Probably because neonates with BA are the minority, little is known about the clinical courses of these during neonatal period.

The diagnostic process of BA still remains challenging especially in neonates. ${ }^{7}$ Preterm infants and babies with various disorders sometimes present with intrahepatic obstructive jaundice. And, the other differential diagnoses including congenital metabolic disease and hepatitis should be considered, but it commonly takes time to obtain the result of the specific examinations. Furthermore, the fact that invasive contrast study is necessary for definite diagnosis of BA also annoys the clinicians.

The present study was designed to review the blood biochemical values of infants with BA mainly during neonatal period, in the 3 tertiary hospitals in Chiba area. Intriguing decreasing trend of direct bilirubin (D-Bil) observed in this study may be useful data for clinicians treating icteric infants.

\section{Materials and Methods}

Nineteen patients with BA ( $15.8 \%$ of total) satisfying following 3 selection criteria were studied: i) patients who underwent hepatoportoenterostomy in Chiba University Hospital (institution A) from August 1988 to December 2012, in Chiba Children's Hospital (institution B) from April 1995 to December 2012, or in Matsudo City Hospital Children's Medical Center (institution C) from May 1986 to December 2012, ii) patients underwent blood tests including direct bilirubin within the first 20 days of age, iii) patients had underwent blood tests including D-Bil at least 2 times within the first 30 days of age. All the diagnoses of BA were confirmed by intraoperative findings. Difference of observation periods between 3 institutions results from difference of date of foundation. Clinical records were reviewed retrospectively. The blood samples collected preoperatively for the clinical purpose were also analyzed.

Results are presented as mean \pm standard deviation. Differences of levels between groups were compared with the student t-test or Welch's t-test. Curves of native liver survival were calculated using Kaplan-Meier methodology and log rank test was used to compare survival rates. $P$ values $<0.05$ were considered significant.
Correspondence: Keita Terui, Department of Pediatric Surgery, Graduate School of Medicine, Chiba University, 1-8-1, Inohana, Chuou-ku, Chiba 260-8677, Japan.

Tel. +81.043.222.7171 - Fax +81.043.2262366

E-mail: kta@cc.rim.or.jp

Key words: biliary atresia, bilirubin, hyperbilirubinemia, neonate, jaundice.

Contributions: KT performed the statistical analysis and wrote the manuscript; YH and ES collected data; TS, TM, MN and JI provided medical care including surgical procedure; and HY designed the study and reviewed the manuscript. All the authors have read and approved the final manuscript.

Conflict of interests: the authors declare no potential conflict of interests.

Received for publication: 23 July 2013

Revision received: 9 October 2013.

Accepted for publication: 7 November 2013.

This work is licensed under a Creative Commons Attribution NonCommercial 3.0 License (CC BYNC 3.0).

(C) Copyright K. Terui et al., 2013

Licensee PAGEPress, Italy

Pediatric Reports 2013; 5:e17

doi:10.4081/pr.2013.e17

\section{Results}

Table 1 shows demographic data of the subjects. They were born weighing $2904 \pm 416$ (1798-3555) g at the $38.9 \pm 2.4$ (34-41) weeks of gestation. Two low-birth-weight babies weighing $1798 \mathrm{~g}$ and $2405 \mathrm{~g}$ were included. The type of BA consisted of type $3(n=16)$ and type 1 $(n=3) .8$ There were 3 cases classified in biliary atresia splenic malformation syndrome (BASM) defined as associated splenic malformation.

The first blood sample of each patient was collected at $8.4 \pm 6.5(0-20)$ days of age. The reasons why the blood samples were collected at an early point consist of typical symptom of BA [icterus $(n=6)$, acholic stool $(n=7)]$, other medical problems including respiratory problem $(n=2)$, intestinal obstruction $(n=2)$, low birth weight $(\mathrm{n}=1)$, and maternal problem $(n=1)$. The acholic stool was obviously observed at age $8.8 \pm 9.0$ (1-31) days. In 16 cases, acholic stools were detected within 2 weeks of age $(5.3 \pm 3.2$ day of age), and over 2 weeks of age ( $27.7 \pm 4.2$ day of age) in the other 3 cases.

Hepatoportoenterostomy was performed at age of $43.2 \pm 18.2$ (23-100) days. Critical complication was seen in 1 case, who underwent 
surgery at 45 days of age and died for uncontrollable postoperative bleeding 3 days after surgery. Redo operation intended to scratch the hepatic portal region again was performed in 4 patients. In these, successful drainage was achieved in 2 cases, but insufficient in 2 cases. In the 14 cases without the perioperative complication nor the redo surgery, the status of jaundice-free (defined as serum levels of total bilirubin $<1.5 \mathrm{mg} / \mathrm{dL}$ within 3 months postoperatively) was accomplished in 12 cases $(85.7 \%)$. Native liver survival of the patients who underwent surgery within 30 days of age was $100 \%$, but had no significant difference compared to the patients with the surgery over 31 days of age $(\mathrm{P}=0.058)$.

The levels of total bilirubin (T-Bil), D-Bil, alanine aminotransferase (ALT) and gammaglutamyl transpeptidase (gGTP) during the first 63 days were shown in Figure 1. Each point represents the average with standard deviation of all the data collected around the 7 days. For instance, the first plot represents the average of all the data collected from multiple patients between 0 and 6 days of age.

Decrease of T-Bil was confirmed in all the subjects, from $10.4 \pm 7.5$ (1-26) to $29.8 \pm 9.1$ (1652 ) days of age, with duration of $20.4 \pm 9.6$ (436) days. The range of reduction was $6.5 \pm 3.3$ (1.1-11.3) $\mathrm{mg} / \mathrm{dL}$, and the reduction rate was $0.36 \pm 0.18(0.08-0.73) \mathrm{mg} / \mathrm{dL} /$ day.

Decrease of D-Bil was confirmed in 17 out of 19 cases (89\%). Decrease of D-Bil was observed from $15.5 \pm 8.0$ (5-40) to $24.9 \pm 9.6$ (943) days of age, with duration of $10.4 \pm 7.0$ (326) days. The range of reduction was $1.1 \pm 1.0$ $(0.2-4.1) \mathrm{mg} / \mathrm{dL}$, and the reduction rate was $0.15 \pm 0.12(0.03-0.53) \mathrm{mg} / \mathrm{dL} /$ day.

A significant decrease of D-Bil was observed in 2 cases (Figure 2). The level of D-Bil continued to decrease for 26 days (from 5 days of age) in case $A$, and 23 days (from 17 days of age) in case B. And, the range of reduction was 2.0 and $4.1 \mathrm{mg} / \mathrm{dL}$, respectively. Interestingly, both the 2 cases are classified in BASM.

\section{Discussion}

BA is an inflammatory, progressive fibrosclerosing cholangiopathy of infancy. ${ }^{1}$ If untreated, cirrhotic change with portal hypertension and liver failure progresses, resulting in death within 2 to 3 years. Even If treated appropriately by hepatoportoenterostomy, however, progressive disease status can not be stopped completely. This knowledge about progressive disease status possibly misleads us to think that D-Bil, practical indicator of obstructive jaundice seems to be progressive as well. The present study showed that $89 \%$ of the subjects had declining trend of D-Bil during $3^{\text {rd }}$ weeks of age. This tendency of D-Bil seems to be along with decrease of T-Bil, meaning that the level of D-Bil in infants of BA can be influenced by normalization of physiological jaun- dice. Clinicians treating icteric infants should keep this acknowledge in mind, and should not exclude a diagnosis of BA even if the level of DBil has declining tendency.

Concept that BA has pathophysiological heterogeneity is being accepted; one is embryonic (fetal, prenatal) type and the other is perinatal (acquired) type. ${ }^{9}$ Although definition of embryonic type BA has not been established yet, at least patients as follows are probably thought to be classified in this category; i) patients with antenatal detection of the abnormal bile duct, ii) BASM, iii) early-onset patients who develop both acholic stool and cholestatic jaundice before 2 weeks of age. ${ }^{10}$ In our series, 16 cases (84\%) had acholic stool within 2 weeks of age. they included 3 cases of BASM, and seemed to be categorized as embryonic type.

Intriguingly, all the cases of embryonic type had declining trend of D-Bil, especially in the cases of BASM. On the other hand, declining trend of D-Bil was not seen in 2 of 3 cases considered of perinatal type. Although the reason why embryonic type is more likely to have declining tendency of D-Bil is unexplainable,

Table 1. Demographic data of 19 subjects.

\begin{tabular}{ll}
\hline Male & $5 / 19(26.3 \%)$ \\
Birth weight & $2904 \pm 416(1798-3555) \mathrm{g}$ \\
\hline Gestation age & $38.9 \pm 2.4(34-41)$ weeks \\
Type 1 : type 3 & $3: 16(15.8 \%)$ \\
\hline Biliary atresia splenic malformation syndrome & $3 / 19(15.8 \%)$ \\
Acholic stool detection & $8.8 \pm 9.0(1-31)$ days \\
\hline Hepatoportoenterostomy & $43.2 \pm 18.2(23-100)$ days of age \\
Redo operation & $4 / 19(21.0 \%)$ \\
\hline
\end{tabular}

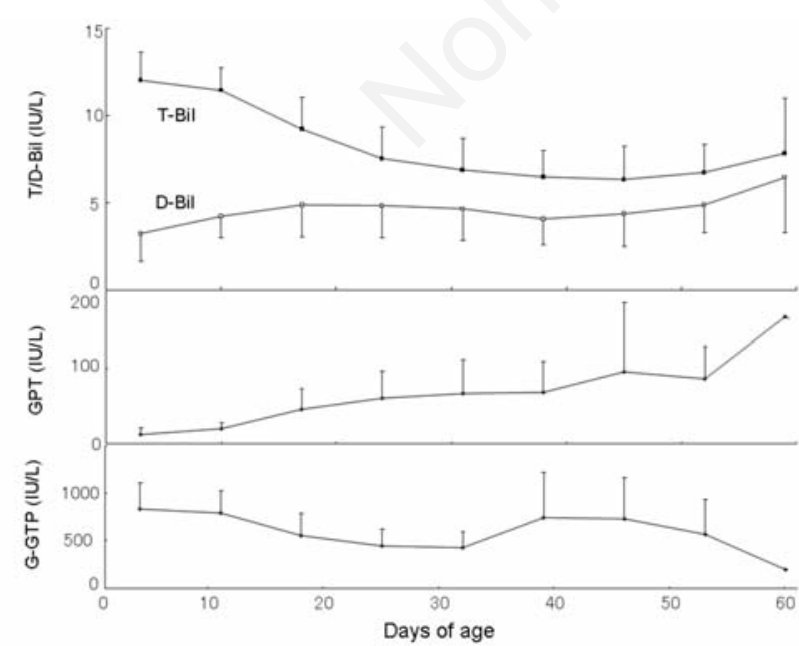

Figure 1. The levels of total bilirubin (T-Bil), direct bilirubin (DBil), alanine aminotransferase (ALT) and gamma-glutamyl transpeptidase (gGTP). Each point represents the average with standard deviation of all the data collected around the 7 days.

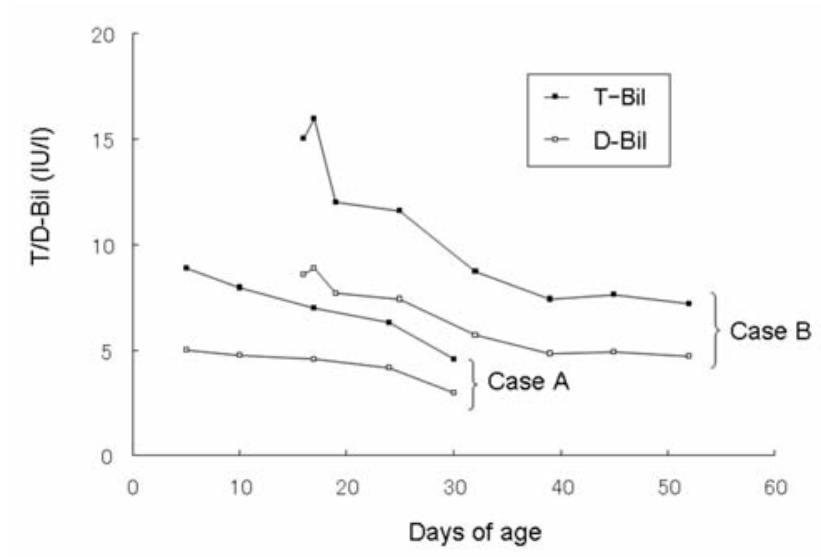

Figure 2. The levels of total bilirubin (T-Bil), direct bilirubin (DBil) of 2 cases with BASM who presented extreme decreasing trend. 
our data supports the pathophysiological hypothesis of heterogeneity from the aspects of clinical course during the neonatal period.

Early presentation of the specific symptoms and accompanied abnormalities may have facilitated earlier detection of embryonic type BA. In contrast, only 3 cases (16\%) considered to be perinatal type BA were included in the present study. This suggests that it is difficult to distinguish perinatal type BA during neonatal period, mainly because acholic stool is absent. All these 3 cases had had abnormally high level of D-Bil before acholic stool was detected. To detect patients with perinatal type BA earlier, therefore, new screening system using sampling of D-Bil, not the infant stool color card is necessary. ${ }^{11}$

The strategy of early operation becomes a broad consensus on treatment of BA. However, appropriate timing of surgery for neonates with BA still remains unclear. Volpert et al. reported worse outcome of patients who underwent the procedure before 30 days of age. ${ }^{12}$ The reason why the earlier surgery resulted in worse outcome was explained by the different pathogenesis of these BA patients that brings it to clinical attention earlier. In contrast, Canadian Pediatric Hepatology Research Group reported better native liver survival in patients who underwent surgery within 30 days of age (49\%), compared to those with surgery between 31 and 90 day of age (36\%). ${ }^{13}$ According to Japanese BA Registry data (1989-2006, $\mathrm{n}=2130)$, rate of postoperative jaundice-free was $65 \%$ in patients who underwent surgery within 30 days, and $58 \%$ in patients who underwent surgery between 41 and 60 days of age. ${ }^{4}$ The most recent data from French group revealed better outcome (both clearance of jaundice and survival with native liver) in patients who received hepatoportoenterostomy in the first month of life compared to patients in the second month of life. ${ }^{14}$ In our series, patients with early surgery ( $<30$ days of age) had better native liver survival, although difference was not significant due to small samples. Considering these facts, early surgery within 30 days is at least not a contraindication, but further study is necessary to elucidate the appropriate timing of surgery for neonates with BA.

\section{Conclusions}

Patients with BA who were examined during neonatal period mainly consisted of cases with embryonic type BA. They had declining tendency of D-Bil during $3^{\text {rd }}$ weeks of age, in association with normalization of physiological jaundice. When clinicians treating icteric infants see this phenomenon, diagnosis of BA should not be excluded.

\section{References}

1. Hartley JL, Davenport M, Kelly DA. Biliary atresia. Lancet 2009;374:1704-13.

2. Muraji T. Early detection of biliary atresia: past, present \& future. Expert Rev Gastroenterol Hepatol 2012;6:583-9.

3. Japanese Biliary Atresia Registry. Results of 1990. J Jpn Soc Pediatr Surg 1992;28: 113-8.
4. Japanese Biliary Atresia Registry. Results of 2006. J Jpn Soc Pediatr Surg 2008;44: 167-76.

5. Wadhwani SI, Turmelle YP, Nagy R, et al. Prolonged neonatal jaundice and the diagnosis of biliary atresia: a single-center analysis of trends in age at diagnosis and outcomes. Pediatrics 2008;121:e1438-40.

6. Karrer FM, Lilly JR, Stewart BA, et al. Biliary atresia registry, 1976-1989. J Pediatr Surg 1990;25:1076-80

7. De Bruyne R, Van Biervliet S, Vande Velde $\mathrm{S}$, et al. Clinical practice: neonatal cholestasis. Eur J Pediatr 2011;170:279-84.

8. Kasai M, Sawaguchi M, Akiyama T. A proposal of a new classification of biliary atresia. J Jpn Soc Pediatr Surg 1976;12:327-31.

9. Shalaby A, Makin E, Davenport M. Classification of the biliary atresia phenotype. Pediatr Int 2010;52:897.

10. Yang MC, Chang MH, Chiu SN, et al. Implication of early-onset biliary atresia and extrahepatic congenital anomalies. Pediatr Int 2010;52:569-72.

11. Harpavat S, Finegold MJ, Karpen SJ. Patients with biliary atresia have elevated direct/conjugated bilirubin levels shortly after birth. Pediatrics 2011;128:e1428-33.

12. Volpert D, White F, Finegold MJ, et al. Outcome of early hepatic portoenterostomy for biliary atresia. J Pediatr Gastro enterol Nutr 2001;32:265-9.

13. Schreiber RA, Barker CC, Roberts EA, et al. Biliary atresia: the Canadian experience. J Pediatr 2007;151:659-65.

14. Chardot C, Buet C, Serinet MO, et al. Improving outcomes of biliary atresia: French national series 1986-2009. J Hepatol. 2013;58:1209-17. 